\title{
Renoprotective effects of olmesartan medoxomil on diabetic nephropathy in streptozotocin-induced diabetes in rats
}

\author{
XIAOFEI SI ${ }^{1}$, PENG LI $^{2}$, YAN ZHANG $^{2}$, YAN ZHANG $^{3}, \mathrm{WEI} \mathrm{LV}^{2}$ and DONG QI ${ }^{2}$ \\ ${ }^{1}$ School of Pharmacy, Shanghai Jiao Tong University, Shanghai 200240; ${ }^{2}$ Yantai Yu Huang Ding Hospital, Yantai, \\ Shandong 264000; ${ }^{3}$ Yantai Hospital of Traditional Chinese Medicine, Yantai, Shandong 264005, P.R. China
}

Received May 9, 2013; Accepted July 11, 2013

DOI: $10.3892 /$ br.2013.183

\begin{abstract}
Olmesartan medoxomil (OM) is one of the newest members of the angiotensin receptor blocker (ARB) family. The renoprotective effects of the angiotensin II type 1 receptor antagonist OM was investigated in a streptozotocin (STZ)-induced diabetic rat model. In this study, we investigated whether OM was able to ameliorate diabetic nephropathy (DN). Thirty male Sprague Dawley rats were assigned to 3 groups: the non-diabetic (group $A, n=10$ ), the untreated STZ-induced DN control (group B, n=10) and the STZ-induced DN treated with OM (group C, $n=10$ ). Blood pressure (BP) and glucose, creatinine $(\mathrm{Cr})$, blood urea nitrogen (BUN), superoxide dismutase (SOD), malondialdehyde (MDA) microalbumin and urinary protein concentrations were measured. In STZ diabetic rats, BP, glucose, Cr, BUN, MDA and urinary protein levels were significantly increased compared to the non-diabetic control group. OM significantly improved the biological indices in the DN rats. The renal pathological changes were also observed under a light microscope. Our results suggested that OM exerted renoprotective effects on rats with STZ-induced diabetes.
\end{abstract}

\section{Introduction}

It was estimated that the prevalence of diabetes among all age groups worldwide was $2.8 \%$ in 2000 and is likely to be $4.4 \%$ in 2030 (1). The number of individuals with diabetes is likely to increase to 366 million by 2030. Diabetic nephropathy (DN) is a major cause of morbidity and mortality, occurring in $20-40 \%$ of diabetic patients (2). DN is the single leading cause of end-stage renal disease (ESRD) $(3,4)$. The incidence of ESRD is a growing problem in all countries with a western lifestyle (5). Hypertension occurs in $~ 50 \%$ of type II diabetes patients and is also a major factor leading to arterial damage.

Correspondence to: Dr Dong Qi, Yantai Yu Huang Ding Hospital, 20 Yudong Road, Yantai, Shandong 264000, P.R. China

E-mail: qidong8028@sina.com

Key words: renoprotective effects, olmesartan medoxomil, diabetic nephropathy
The resulting arterial damage is usually progressive and accelerates the development of DN and ESRD (6).

The renin-angiotensin-aldosterone system (RAAS) is crucial in the control of blood pressure (BP) and the pathogenesis of hypertension (7). Blocking the activity of the RAAS is extensively used in the management of hypertension. The renal protective effects of angiotensin II type 1 (AT1) receptor blockers (ARBs) have been demonstrated in animal models of diabetes, including type 1 and 2 diabetic rats $(8,9)$. Olmesartan medoxomil (OM) is one of the newest additions to the ARB family and it may be rapidly and completely de-esterified to olmesartan following oral administration. To the best of our knowledge, the ability of OM to control DN in animal models of streptozotocin (STZ)-induced diabetes has not been investigated, although OM was previously demonstrated to retard the progression of DN (10). The utilization of OM in this STZ-induced diabetes animal model appears promising in elucidating the mechanism underlying DN and advancing translational research $(11,12)$. The purpose of this study was to evaluate the efficacy of OM in the treatment of DN by investigating the renoprotective effects of this drug in an STZ-induced diabetes rat model.

\section{Materials and methods}

Chemicals and instruments. OM was supplied by the Shanghai Sankyo Pharmaceutical Co., Ltd. (Shanghai, China). The standard STZ was purchased from Sigma Chemical Co. (St. Louis, MO, USA). Creatinine ( $\mathrm{Cr}$ ), blood urea nitrogen (BUN), superoxide dismutase (SOD), malondialdehyde (MDA) and protein test kits were purchased from the Nanjing Jiancheng Bioengineering Institute (Nanjing, China). All other chemicals and reagents used were of analytical grade.

Animals. Thirty male Sprague Dawley rats, weighing 180-240 g, were purchased from the Experimental Animal Center of Luye Pharmaceutical Company [certificate no. SCXK (Lu) 20030008]. The rats were kept in a room at a relative humidity of $55 \%$ (permissible range: $30-70 \%$ ) and a temperature of $23^{\circ} \mathrm{C}$ (permissible range: $20-26^{\circ} \mathrm{C}$ ) under a 12-h light/dark cycle. The rats were allowed free access to food and water.

All the experiments in this study were conducted in accordance with the Guidelines for the Care and Use of Laboratory 
Table I. Changes in body and renal weight.

\begin{tabular}{|c|c|c|c|c|}
\hline \multirow[b]{2}{*}{ Groups } & \multicolumn{3}{|c|}{ Body weight (g) } & \multirow{2}{*}{$\begin{array}{c}\text { Renal weight } \\
\text { (g/100 g body weight) }\end{array}$} \\
\hline & Initial & Final & Gain (12 weeks) & \\
\hline A (normal) & $265.8 \pm 7.5$ & $360.1 \pm 14.1$ & $95.2 \pm 7.8$ & $0.35 \pm 0.04$ \\
\hline B (DN control) & $252.3 \pm 5.4^{\mathrm{b}}$ & $271.5 \pm 6.3^{b}$ & $19.2 \pm 5.6^{\mathrm{b}}$ & $0.71 \pm 0.05^{\mathrm{b}}$ \\
\hline $\mathrm{C}(\mathrm{OM})$ & $256.9 \pm 6.9^{a, b}$ & $304.8 \pm 5.7^{\mathrm{a}, \mathrm{b}}$ & $48.9 \pm 6.3^{\mathrm{a}, \mathrm{b}}$ & $0.56 \pm 0.03^{\mathrm{a}, \mathrm{b}}$ \\
\hline
\end{tabular}

${ }^{\mathrm{a}} \mathrm{P}<0.05$ vs. DN control values; ${ }^{\mathrm{b}}<0.05$ vs. normal values. $\mathrm{DN}$, diabetic nephropathy; $\mathrm{OM}$, olmesartan medoxomil.

Table II. Effects of OM on blood pressure and biological indices in serum and urine.

\begin{tabular}{|c|c|c|c|}
\hline Variables & A (normal) & $\mathrm{B}$ (DN control) & $\mathrm{C}(\mathrm{OM})$ \\
\hline $\mathrm{BP}(\mathrm{mmHg})$ & $100.25 \pm 1.91$ & $118.12 \pm 2.13^{\mathrm{b}}$ & $102.18 \pm 2.42^{\mathrm{a}, \mathrm{b}}$ \\
\hline \multicolumn{4}{|l|}{ Serum } \\
\hline Glucose (mmol/l) & $9.00 \pm 0.35$ & $34.40 \pm 0.75^{\mathrm{b}}$ & $22.00 \pm 0.26^{\mathrm{a}, \mathrm{b}}$ \\
\hline Albumin $(\mathrm{g} / \mathrm{dl})$ & $35.65 \pm 0.45$ & $30.32 \pm 0.26^{\mathrm{b}}$ & $31.84 \pm 0.21^{\mathrm{a}, \mathrm{b}}$ \\
\hline Creatinine $(\mu \mathrm{mol} / \mathrm{l})$ & $7.43 \pm 2.01$ & $78.08 \pm 1.95^{\mathrm{b}}$ & $61.45 \pm 1.83^{\mathrm{a}, \mathrm{b}}$ \\
\hline BUN (mmol/l) & $9.35 \pm 0.37$ & $18.97 \pm 1.31^{\mathrm{b}}$ & $14.28 \pm 0.97^{\mathrm{a}, \mathrm{b}}$ \\
\hline SOD (U/mg prot) & $1.20 \pm 0.05$ & $0.98 \pm 0.02^{b}$ & $1.11 \pm 0.03^{\mathrm{a}, \mathrm{b}}$ \\
\hline $\operatorname{MDA}(\mathrm{nmol} / \mathrm{ml})$ & $3.01 \pm 0.27$ & $5.76 \pm 0.31^{b}$ & $4.31 \pm 0.28^{a, b}$ \\
\hline \multicolumn{4}{|l|}{ Urine } \\
\hline $\mathrm{CCr}(\mathrm{ml} / \mathrm{kg}$ body weight/min) & $25.45 \pm 1.23$ & $16.94 \pm 1.38^{\mathrm{b}}$ & $20.95 \pm 0.89^{\mathrm{a}, \mathrm{b}}$ \\
\hline Protein excretion (mg/day) & $8.34 \pm 0.61$ & $43.26 \pm 5.93^{\mathrm{b}}$ & $23.83 \pm 3.34^{\mathrm{a}, \mathrm{b}}$ \\
\hline mALB $(\mathrm{ng} / \mathrm{ml})$ & $16.53 \pm 0.54$ & $35.04 \pm 0.38^{\mathrm{b}}$ & $28.93 \pm 0.86^{\mathrm{a}, \mathrm{b}}$ \\
\hline
\end{tabular}

${ }^{\mathrm{a}} \mathrm{P}<0.05$ vs. DN control values; ${ }^{\mathrm{b}} \mathrm{P}<0.05$ vs. normal values. OM, olmesartan medoxomil; BP, blood pressure; BUN, blood urea nitrogen; SOD, superoxide dismutase; MDA, malondialdehyde; $\mathrm{CCr}$, creatinine clearance; mALB, microalbumin.

Animals of Yantai University and were approved by the Animal Study Committee.

Experimental design. Following several days of acclimatization, the rats $(n=30)$ were injected intraperitoneally with STZ dissolved in citrate buffer ( $\mathrm{pH} 4.5$ ) at a dose of $65 \mathrm{mg} / \mathrm{kg}$ body weight. After 3 days, induction of diabetes was confirmed by measuring blood glucose concentration ( $\geq 16.7 \mathrm{mM})(13)$. The rats with blood glucose levels $>16.7 \mathrm{mM}$ were randomly divided into 2 groups. One group was used as the DN control $(n=10)$ and the other group $(n=10)$ received OM at a dose of $10 \mathrm{mg} / \mathrm{kg}$ body weight/day via oral gavage. A normal group of rats $(n=10)$ that underwent sham operation was also included. During the course of the experiment, the normal and control groups received physiological saline of equal volumes. The serum glucose was measured at $0,4,8$, and 12 weeks after overnight fasting. At 12 weeks, 12-h urine samples were collected using metabolic cages and the rats were weighed and sacrificed. Blood samples were obtained from the abdominal aorta. The serum was immediately separated from the blood by centrifugation at $1,600 \times \mathrm{g}$ for $25 \mathrm{~min}$ at $4^{\circ} \mathrm{C}$ and stored at $-80^{\circ} \mathrm{C}$. The left kidneys were removed and weighed following renal perfusion through the renal artery with ice-cold physiological saline $(10,14)$. After rinsing with phosphate-buffered saline, kidney sections were sliced and immersed in 10\% formalin for histological evaluation, whereas the remaining sections were frozen at $-80^{\circ} \mathrm{C}$.

Biochemical measurement. The plasma samples were used for the measurement of glucose, $\mathrm{Cr}$ concentration, BUN, SOD, MDA and albumin. The indices were examined with an ultraviolet-visible spectrophotometer using commercial reagents. Urine samples were collected using metabolic cages and the supernatant was used for examination of the urinary protein concentration. The $\mathrm{Cr}$ clearance $(\mathrm{CCr})$ was calculated using the equation: $\mathrm{CCr}(\mathrm{ml} / \mathrm{kg}$ bw $/ \mathrm{min})=\left[\mathrm{U}_{\mathrm{Cr}}\right.$ $(\mathrm{mg} / \mathrm{dl}) \times$ urine volume $\left.(\mathrm{ml}) / \mathrm{S}_{\mathrm{Cr}}(\mathrm{mg} / \mathrm{dl})\right]$ x $[1000 / \mathrm{bw}$ $(\mathrm{g})] \mathrm{x}[1 / 720(\mathrm{~min})]$, where $\mathrm{U}_{\mathrm{Cr}}$ is the urinary creatinine, $\mathrm{S}_{\mathrm{Cr}}$ is the serum creatinine and bw is the body weight. The levels of microalbumin (mALB) were quantified by ELISA (YfSwBio Shanghai, China).

Histological analysis. Renal tissues were fixed in $10 \%$ formalin solution and embedded in paraffin. Sections $(2 \mu \mathrm{m})$ were obtained by a Polycut microtome (CM1950; Leica, Mannheim, Germany) and stained with hematoxylin and 
eosin. The sections were then examined under a light microscope.

Statistical analysis. Data are expressed as means \pm standard deviation. Significant differences within the groups were calculated by one-way ANOVA. Statistical differences between groups were evaluated by the Student's unpaired t-test, using SPSS statistical software, version 11.5 (SPSS Inc., Chicago, IL, USA). $\mathrm{P}<0.05$ was considered to indicate a statistically significant difference.

\section{Results}

Body and kidney weight changes. The effects of OM administered by oral gavage on the body and kidney weight of the rats are presented in Table I. The body weights of the rats in the treatment group was increased compared to that of the DN rats. Furthermore, the body weight gain of normal rats was 4.95 times higher compared to the DN control rats. In the treatment group, there was a 1.94-fold increase in the body weight. However, the kidney weight of $\mathrm{DN}$ control rats was 2.0 times higher compared to the normal group, indicating reduced enlargement due to $\mathrm{OM}$ administration.

Serum indices and BP. As shown in Table II, the administration of $\mathrm{OM}$ affected serum glucose and albumin levels to a certain extent. The serum glucose level in the DN control group was $34.40 \mathrm{mmol} / \mathrm{l}$, which was significantly higher compared to the normal group. However, the serum glucose level decreased to $22.00 \mathrm{mmol} / 1$ following administration of OM. The serum albumin level in the DN control rats was $30.32 \mathrm{~g} / 1$, which was lower compared to the normal rats. These levels were significantly increased by $5.0 \%$ following treatment with OM. The changes in blood glucose during 12 weeks are shown in Fig. 1. The level of hyperglycemia was significantly reduced by OM at 12 weeks. However, at 4 and 8 weeks there was only a minor decrease in hyperglycemia.

The serum SOD concentration in DN rats was lower compared to that in normal rats and the SOD concentration was increased by $13.27 \%$ with OM treatment. The serum MDA level was increased by 1.91 -fold in DN rats and decreased with OM treament. Over the course of this study, BP was elevated in DN compared to normal rats and treatment with OM lowered BP to almost normal levels (Table II).

Parameters of renal function. The effects of OM on serum and urinary parameters of renal function are presented in Table II. BUN, urinary protein excretion and serum $\mathrm{Cr}$ levels were higher in the DN control compared to the normal group. Following oral administration of OM, the BUN and serum $\mathrm{Cr}$ levels were decreased. In addition, the use of OM decreased urinary protein excretion from 43.26 to $23.83 \mathrm{mg}$ /day. The urinary $\mathrm{mALB}$ concentration was increased significantly in $\mathrm{DN}$ rats and was decreased significantly with $\mathrm{OM}(\mathrm{P}<0.05)$.

Histopathological changes. Light microscopy examination revealed lesions and an increase of the mesangial matrix in the DN control group. In addition, glomerular hypertrophy in the DN group was more prominent compared to that observed in normal rats. The OM treatment group exhibited minimal

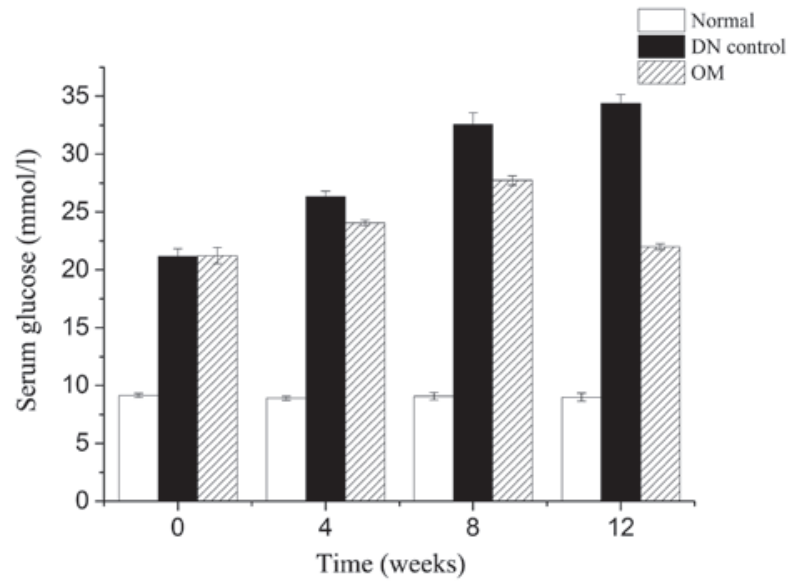

Figure 1. Changes in serum glucose during 12 weeks with oral olmesartan medoxomil (OM) treatment. DN, diabetic nephropathy.

A

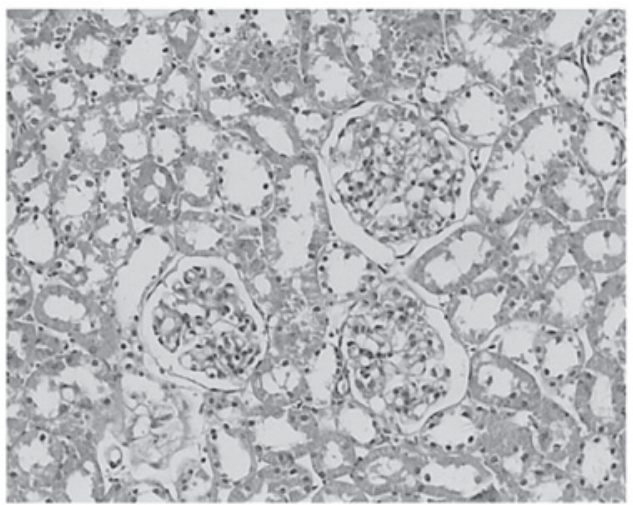

$\mathbf{B}$

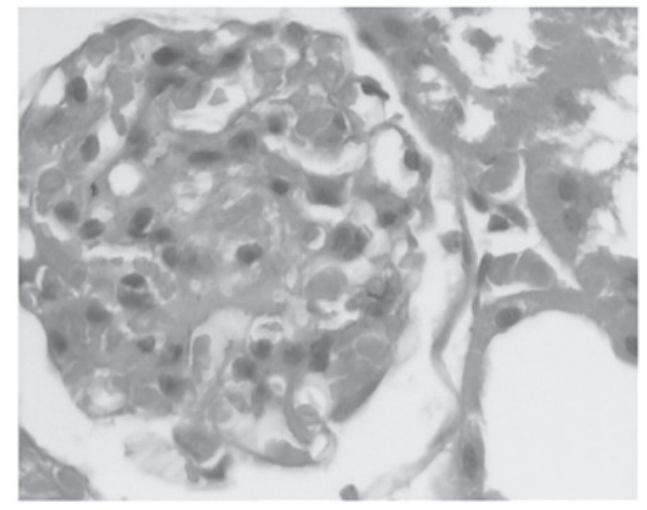

C

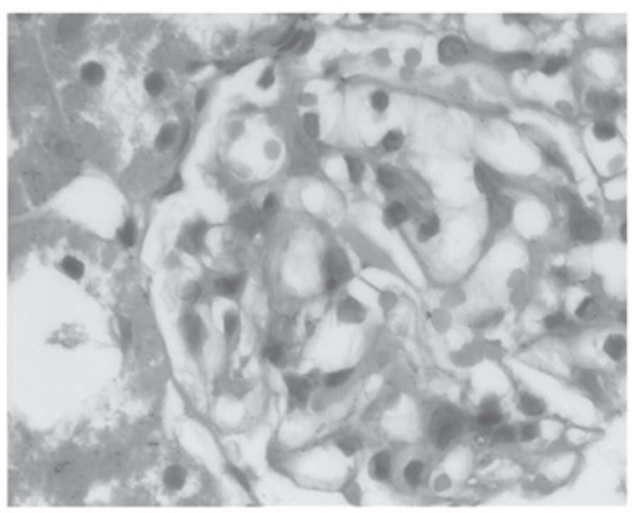

Figure 2. Renal histology. Renal tissue from (A) normal rats, (B) DN control rats and (C) DN rats treated with olmesartan medoxomil. DN, diabetic nephropathy. 
exudative renal lesions compared to those in the DN control group (Fig. 2) and presented with decreased glomerular hypertrophy to a certain extent.

\section{Discussion}

STZ is toxic to pancreatic $\beta$ cells $(15,16)$ and has been widely used to induce diabetes in animal models (17). There are various animal models of diabetes with different origin, characteristics and underlying causes (18). However, STZ-induced experimental diabetes exhibits sustained hyperglycemia and well-characterized diabetic complications with a low incidence of mortality (15). In this study, an STZ-induced diabetes animal model was selected to investigate the ability of OM to control DN.

DN is characterized by excessive amassing of extracellular matrix, with thickening of the glomerular and tubular basement membranes and an increased amount of mesangial matrix (19). The STZ-injected rats exhibited the main characteristics of diabetes mellitus and the changes in the DN markers in our study were similar to those previously reported (20-22). In the present study, OM was able to improve these parameters in DN rats.

Hypertension is one of the most important contributing factors to DN. Therefore, BP needs to be tightly regulated. The angiotensin-converting enzyme inhibitors are a group of drugs that are beneficial in the treatment of DN (23). The angiotensin II (Ang II) receptor blockers (ARBs) have been shown to retard the progression of nephropathy in patients with diabetes. The renoprotective effects of ARBs in rat models were previously reported $(24,25)$. OM, one of the most potent ARBs, was demonstrated to increase plasma renin activity in rats. Therefore, the renoprotective actions demonstrated in this study may be attributed to the systemic and intrarenal blockade of the renin-angiotensin system (26). In this study, it was demonstrated that OM was able to decrease BP. It was also demonstrated that the serum glucose concentration in diabetic rats was by 3.78-fold higher compared to that in normal rats and treatment with OM significantly inhibited hyperglycemia. $\mathrm{OM}$ also reduced renal weight and increased body weight. The mechanism of the inhibition of hyperglycemia by OM has not been elucidated, although it may be through the blockade of RAS or the inhibition of oxidative stress.

The common characteristic in the development of DN is the decrease in the glomerular filtration rate, which may reflect serum $\mathrm{Cr}$ and $\mathrm{CCr}$ levels and lead to proteinuria. The DN rats exhibited significant increases in serum $\mathrm{Cr}$, BUN and urinary protein excretion, whereas the $\mathrm{CCr}$ level was significantly decreased compared to that in normal rats. According to a previous study by Oktem et al (22), proteinuria is an important indicator of early-stage DN and accelerates the occurrence of tubular cell damage. Another previous study (27) reported that $\mathrm{mALB}$ is a sensitive and specific predictor of DN and it has been widely used as a clinical index of early-stage DN. ARBs were reported to reduce proteinuria in a number of animal models of diabetes $(9,28)$. OM significantly inhibited the development of mALB in DN rats. The increasing levels of BUN and serum $\mathrm{Cr}$ may indicate progressive renal damage and the present study demonstrated that OM positively affected these parameters.
It was previously demonstrated that increased oxidative stress and reactive oxygen species are involved in the pathogenesis of diabetes (29). Oxidative stress may increase the production of free oxygen radicals, promote the formation of lipid peroxidation products and reduce the level of antioxidant enzymes, such as SOD. SOD is a scavenger of free radicals and the protective effect of SOD on renal function is directly associated with its ability to alleviate oxidative stress. MDA is an end-product of lipid peroxidation and may reflect the degree of oxidation in renal tissues. OM was reported to improve endothelin-induced hypertension and oxidative stress in rats (10). According to Fujimoto et al (30), OM may inhibit superoxide production and oxidative stress, independent of its BP-lowering effect. The data in our study demonstrated that the serum SOD concentrations in diabetic rats were lower compared to those in normal rats and the levels of SOD were increased, whereas MDA levels were decreased following treatment with OM.

In conclusion, our results suggest that OM exerts a beneficial effect on DN via different pathways and it may be a potential renoprotective pharmaceutical for the treatment of DN.

\section{Acknowledgements}

This study was supported by the Programs for Science and Technology Development and Plan of Yantai (no. 2012076) and Science Foundation for The Excellent Youth Scholars of Shandong Province (no. 2008BS02027).

\section{References}

1. Wild S, Roglic G, Green A, Sicree R and King H: Global prevalence of diabetes: estimates for the year 2000 and projections for 2030. Diabetes Care 27: 1047-1053, 2004.

2. Tan AL, Forbes JM and Cooper ME: AGE, RAGE, and ROS in diabetic nephropathy. Semin Nephrol 27: 130-143, 2007.

3. Dalla Vestra M, Saller A, Bortoloso E, Mauer M and Fioretto P: Structural involvement in type 1 and type 2 diabetic nephropathy. Diabetes Metab 26 (Suppl 4): 8-14, 2000.

4. American Diabetes Association: Standards of medical care in diabetes. Diabetes Care 28 (Suppl 1): 4-36, 2005.

5. Ritz E, Rychlik I, Locatelli F and Halimi S: End-stage renal failure in type 2 diabetes: a medical catastrophe of worldwide dimensions. Am J Kidney Dis 34: 795-808, 1999.

6. Pugsley MK: The angiotensin-II (AT-II) receptor blocker olmesartan reduces renal damage in animal models of hypertension and diabetes. Proc West Pharmacol Soc 48: 35-38, 2005.

7. de Gasparo M, Catt KJ, Inagami T, Wright JW and Unger T: International union of pharmacology. XXIII. The angiotensin II receptors. Pharmacol Rev 52: 415-472, 2000.

8. Kohzuki M, Yasujima M, Kanazawa M, et al: Antihypertensive and renal-protective effects of losartan in streptozotocin diabetic rats. J Hypertens 13: 97-103, 1995.

9. Uehara Y, Hirawa N, Kawabata Y, et al: Angiotensin II subtype-1 receptor antagonists improve hemodynamic and renal changes without affecting glucose metabolisms in genetic rat model of non-insulin-dependent diabetes mellitus. Am J Hypertens 12: 21-27, 1999

10. Yao L, Kobori H, Rahman M, et al: Olmesartan improves endothelin-induced hypertension and oxidative stress in rats. Hypertens Res 27: 493-500, 2004.

11. Adams AJ: Bench to bedside and bedside to bench: where are we? Optom Vis Sci 83: 125-126, 2006.

12. Kelly A, Li C, Gao Z, Stanley CA and Matschinsky FM: Glutaminolysis and insulin secretion: from bedside to bench and back. Diabetes 51 (Suppl 3): 421-426, 2002.

13. Dai GL, He JK, Xie Y, Han R, Qin ZH and Zhu LJ: Therapeutic potential of Naja naja atra venom in a rat model of diabetic nephropathy. Biomed Environ Sci 25: 630-638, 2012. 
14. Yamabe N, Yokozawa T, Oya $\mathrm{T}$ and Kim M: Therapeutic potential of (-)-epigallocatechin 3-O-gallate on renal damage in diabetic nephropathy model rats. J Pharmacol Exp Ther 319: 228-236, 2006

15. Ozturk Y, Altan VM and Yildizoglu-Ari N: Effects of experimental diabetes and insulin on smooth muscle functions. Pharmacol Rev 48: 69-112, 1996.

16. Junod A, Lambert AE, Orci L, Pictet R, Gonet AE and Renold AE: Studies of the diabetogenic action of streptozotocin. Proc Soc Exp Biol Med 126: 201-205, 1967.

17. Jones RB, Dickinson K, Anthony DM, Marita AR, Kaul CL and Buckett WR: Evaluation of BTS 67582 , a novel antidiabetic agent, in normal and diabetic rats. Br J Pharmacol 120: 1135-1143, 1997.

18. Srinivasan K and Ramarao P: Animal models in type 2 diabetes research: an overview. Indian J Med Res 125: 451-472, 2007.

19. Kanwar YS, Wada J, Sun L, et al: Diabetic nephropathy: mechanisms of renal disease progression. Exp Biol Med (Maywood) 233: 4-11, 2008.

20. Nakagawa T, Sato W, Glushakova O, et al: Diabetic endothelial nitric oxide synthase knockout mice develop advanced diabetic nephropathy. J Am Soc Nephrol 18: 539-550, 2007.

21. Ota T, Takamura T, Ando H, Nohara E, Yamashita H and Kobayashi K: Preventive effect of cerivastatin on diabetic nephropathy through suppression of glomerular macrophage recruitment in a rat model. Diabetologia 46: 843-851, 2003.

22. Oktem F, Ozguner F, Yilmaz HR, Uz E and Dundar B: Melatonin reduces urinary excretion of $\mathrm{N}$-acetyl-beta-D-glucosaminidase, albumin and renal oxidative markers in diabetic rats. Clin Exp Pharmacol Physiol 33: 95-101, 2006.

23. Lewis EJ, Hunsicker LG, Bain RP and Rohde RD: The effect of angiotensin-converting enzyme inhibition on diabetic nephropathy. The Collaborative Study Group. N Engl J Med 329: $1456-1462,1993$.
24. Noda M, Matsuo T, Fukuda R, et al: Effect of candesartan cilexetil (TCV-116) in rats with chronic renal failure. Kidney Int 56: 898-909, 1999.

25. Ueno T, Kaname S, Takaichi K, et al: LOX-1, an oxidized low-density lipoprotein receptor, was upregulated in the kidneys of chronic renal failure rats. Hypertens Res 26: 117-122, 2003.

26. Zhou A, Yu L, Li J, Zhang J and Wang H: Renal protective effects of blocking the intrarenal renin-angiotensin system: angiotensin II type I receptor antagonist compared with angiotensin-converting enzyme inhibitor. Hypertens Res 23: 391-397, 2000.

27. Tabaei BP, Al-Kassab AS, Ilag LL, Zawacki CM and Herman WH: Does microalbuminuria predict diabetic nephropathy? Diabetes Care 24: 1560-1566, 2001.

28. Inada Y, Murakami M, Tazawa S and Akahane M: KRH-594, a new angiotensin AT1 receptor antagonist, ameliorates nephropathy and hyperlipidaemia in diabetic spontaneously hypertensive rats. Clin Exp Pharmacol Physiol 27: 270-276, 2000.

29. Baynes JW and Thorpe SR: Role of oxidative stress in diabetic complications: a new perspective on an old paradigm. Diabetes 48: 1-9, 1999.

30. Fujimoto S, Satoh M, Horike H, et al: Olmesartan ameliorates progressive glomerular injury in subtotal nephrectomized rats through suppression of superoxide production. Hypertens Res 31: 305-313, 2008. 